\title{
POSITIVE RADIAL SOLUTIONS \\ OF THE DIRICHLET PROBLEM \\ FOR THE MINKOWSKI-CURVATURE EQUATION IN A BALL
}

\author{
Isabel Coelho - Chiara Corsato - Sabrina Rivetti
}

Abstract. We study the existence and multiplicity of positive radial solutions of the Dirichlet problem for the Minkowski-curvature equation

$$
\begin{cases}-\operatorname{div}\left(\frac{\nabla v}{\sqrt{1-|\nabla v|^{2}}}\right)=f(|x|, v) & \text { in } B_{R} \\ v=0 & \text { on } \partial B_{R}\end{cases}
$$

where $B_{R}$ is a ball in $\mathbb{R}^{N}(N \geq 2)$. According to the behaviour of $f=f(r, s)$ near $s=0$, we prove the existence of either one, two or three positive solutions. All results are obtained by reduction to an equivalent non-singular one-dimensional problem, to which variational methods can be applied in a standard way.

\section{Introduction}

This paper focuses on the study of the existence and multiplicity of positive solutions for the quasilinear mixed boundary-value problem

2010 Mathematics Subject Classification. 35J25, 35J60, 35J62, 35J20, 35J93, 47J30.

Key words and phrases. Quasilinear elliptic differential equation, Minkowski-curvature, Dirichlet boundary condition, radial solution, positive solution, existence, multiplicity, variational methods.

Research of the first named author supported by Fundação para a Ciência e a Tecnologia (SFRH/BD/61484/2009).

Research of the second and third named authors supported by MIUR, in the frame of the PRIN "Equazioni differenziali ordinarie e applicazioni", and by University of Trieste, in the frame of the FRA project "Equazioni differenziali ordinarie: aspetti qualitativi e numerici". 


$$
\left\{\begin{array}{l}
\left.-\left(\frac{r^{N-1} u^{\prime}}{\sqrt{1-\left|u^{\prime}\right|^{2}}}\right)^{\prime}=r^{N-1} f(r, u) \text { in }\right] 0, R[, \\
u^{\prime}(0)=0, \quad u(R)=0,
\end{array}\right.
$$

where $N \in \mathbb{N}, N \geq 2$. Solutions of (1.1) correspond to the radially symmetric solutions of the $N$-dimensional Dirichlet problem associated with the Minkowskicurvature equation

$$
\begin{cases}-\operatorname{div}\left(\frac{\nabla v}{\sqrt{1-|\nabla v|^{2}}}\right)=f(|x|, v) & \text { in } B_{R}, \\ v=0 & \text { on } \partial B_{R}\end{cases}
$$

where $B_{R}$ is the open ball centered at 0 of radius $R$ in $\mathbb{R}^{N}$ and $v(x)=u(r)$ for all $x \in \overline{B_{R}}$ with $r=|x|$.

We point out that this problem is of interest in the theory of relativity: for a discussion we refer, e.g. to [2], [10] and the references contained therein. The existence of radial solutions of (1.2) has been recently discussed in [3] and [12]. One further motivation for studying the existence of positive solutions of (1.1) comes from the observation that any positive solution $u \in C^{2}\left(\overline{B_{R}}\right)$ of the autonomous $N$-dimensional problem

$$
\begin{cases}-\operatorname{div}\left(\frac{\nabla v}{\sqrt{1-|\nabla v|^{2}}}\right)=f(v) & \text { in } B_{R}, \\ v=0 & \text { on } \partial B_{R},\end{cases}
$$

with $f \in C^{1}(\mathbb{R})$, is necessarily radially symmetric. This fact is a simple consequence of [11]: some details are given in the Appendix.

This work provides a partial extension of the results obtained in [7] to the radial problem (1.1). It also yields a completion of the recent papers [4] and [5]. Indeed, we are able to consider more general nonlinearities and to get higher multiplicity results.

The model example is

$$
f(r, s)=\lambda a(r) s^{p}+\mu b(r) s^{q} .
$$

Here the exponents $p, q$ satisfy $0<p<1<q$, the parameters $\lambda, \mu$ are nonnegative, and the functions $a, b:[0, R] \rightarrow \mathbb{R}$ are continuous and positive somewhere, in particular they may change sign. Under these assumptions we prove the existence of at least three positive solutions for all sufficiently large values of $\mu$ and all small positive values of $\lambda$. However, other situations can be dealt with. In particular, if $\lambda=0$, we obtain the existence of at least two positive solutions for sufficiently large values of the parameter $\mu$. On the other hand, if $\mu=0$, we can guarantee the existence of at least one positive solution for any $\lambda>0$. Finally, supposing that $f$ is linear, e.g. $\lambda=0$ and $q=1$, we show the existence of at least one positive solution, provided $\mu$ is sufficiently large. 
We remark that, unlike the semilinear case, we can avoid here any growth restriction on $f$ with respect to possible critical exponents.

Similarly to [7], our approach is variational and based upon the search of nontrivial critical points of the action functional associated with a suitable modified problem.

We finally point out that our results here can be effectively used to study the existence of solutions of the problem

$$
\begin{cases}-\operatorname{div}\left(\frac{\nabla v}{\sqrt{1-|\nabla v|^{2}}}\right)=f(x, v, \nabla v) & \text { in } \Omega \\ v=0 & \text { on } \partial \Omega\end{cases}
$$

where $\Omega$ is a general bounded regular domain in $\mathbb{R}^{N}$. This approach is exploited in [8], [9].

This paper is organized as follows. In Section 2 we introduce the space where to settle the equivalent one-dimensional problem and we prove some apriori estimates. Section 3 is devoted to the statement and proof of our existence and multiplicity results.

Notation. We list a few notations that will be used throughout this paper. For functions $u, v:[0, R] \rightarrow \mathbb{R}$ we write $u \geq v$ if $u(r) \geq v(r)$ almost everywhere in $[0, R]$. Instead we write $u>v$ if $u \geq v$ and $u(r)>v(r)$ in a subset of $[0, R]$ having positive measure, moreover we say that $u$ is positive if $u>0$. We also set $u \vee v=\max \{u, v\}$ and $u \wedge v=\min \{u, v\}$. In particular, $u^{+}=u \vee 0$ and $u^{-}=-(u \wedge 0)$. Finally, for $N \in \mathbb{N}, N \geq 2,2^{*}$ denotes $2 N /(N-2)$ (to be read $+\infty$ if $N=2$ ).

\section{Preliminaries}

This section is devoted to the introduction of some technical tools that will be used in the sequel. Throughout we assume:

$\left(\mathrm{h}_{1}\right) f:[0, R] \times \mathbb{R} \rightarrow \mathbb{R}$ satisfies the $L^{1}$-Carathéodory conditions, i.e. for almost every $r \in[0, R], f(r, \cdot): \mathbb{R} \rightarrow \mathbb{R}$ is continuous, for every $s \in \mathbb{R}$, $f(\cdot, s):[0, R] \rightarrow \mathbb{R}$ is measurable, and for each $M>0$ there is $\gamma \in$ $L^{1}(0, R)$ such that $|f(r, s)| \leq \gamma(r)$ for almost every $r \in[0, R]$ and every $s \in[-M, M]$,

and we set

$$
F(r, s)=\int_{0}^{s} f(r, \xi) d \xi .
$$

We define $\phi, \Phi:]-1,1[\rightarrow \mathbb{R}$ by

$$
\phi(y)=\frac{y}{\sqrt{1-y^{2}}} \quad \text { and } \quad \Phi(y)=\int_{0}^{y} \phi(\xi) d \xi .
$$


Notion of solution. We say that a function $u \in C^{1}([0, R])$ is a solution of (1.1) if $\left\|u^{\prime}\right\|_{\infty}<1, r^{N-1} \phi\left(u^{\prime}\right) \in W^{1,1}(0, R), u$ satisfies the equation almost everywhere in $[0, R]$ and the boundary conditions in (1.1). Further, it is said to be positive if $u>0$.

With the aim of finding positive solutions of (1.1), we will start by introducing an equivalent problem. Let us consider a modification of the function $f$. Define $\tilde{f}:[0, R] \times \mathbb{R} \rightarrow \mathbb{R}$ by setting, for almost every $r \in[0, R]$,

$$
\widetilde{f}(r, s)= \begin{cases}0 & \text { if }|s| \geq R+1 \\ f(r, s) & \text { if } 0 \leq s \leq R \\ \text { linear } & \text { if }-(R+1)<s<0 \text { or } R<s<R+1\end{cases}
$$

such that $\widetilde{f}(r, \cdot)$ is continuous for almost every $r \in[0, R]$. We notice that $\widetilde{f}$ satisfies the $L^{1}$-Carathéodory conditions and that there exists $\gamma \in L^{1}(0, R)$ such that

$$
|\widetilde{f}(r, s)| \leq \gamma(r),
$$

for almost every $r \in[0, R]$ and for every $s \in \mathbb{R}$. Set $\sigma=\phi^{\prime}\left(\phi^{-1}\left(\|\gamma\|_{L^{1}}\right)\right)$ and define $\psi: \mathbb{R} \rightarrow \mathbb{R}$ such that

$$
\psi(y)= \begin{cases}\sigma \cdot\left(y+\phi^{-1}\left(\|\gamma\|_{L^{1}}\right)\right)-\|\gamma\|_{L^{1}} & \text { if } y<-\phi^{-1}\left(\|\gamma\|_{L^{1}}\right), \\ \phi(y) & \text { if }|y| \leq \phi^{-1}\left(\|\gamma\|_{L^{1}}\right), \\ \sigma \cdot\left(y-\phi^{-1}\left(\|\gamma\|_{L^{1}}\right)\right)+\|\gamma\|_{L^{1}} & \text { if } y>\phi^{-1}\left(\|\gamma\|_{L^{1}}\right) .\end{cases}
$$

Let $\Psi: \mathbb{R} \rightarrow \mathbb{R}$ be given by

$$
\Psi(y)=\int_{0}^{y} \psi(\xi) d \xi .
$$

It satisfies

$$
\frac{1}{2} y^{2} \leq \Psi(y) \leq \frac{1}{2} \sigma y^{2} \quad \text { for all } y \in \mathbb{R} .
$$

Consider the modified problem

$$
\left\{\begin{array}{l}
\left.-\left(r^{N-1} \psi\left(u^{\prime}\right)\right)^{\prime}=r^{N-1} \widetilde{f}(r, u) \quad \text { in }\right] 0, R[, \\
u^{\prime}(0)=0, \quad u(R)=0 .
\end{array}\right.
$$

We say that a function $u \in C^{1}([0, R])$ is a solution of the equation in (2.7) if $r^{N-1} \psi\left(u^{\prime}\right) \in W^{1,1}(0, R)$ and $u$ satisfies the equation in (2.7) almost everywhere in $[0, R]$; moreover $u$ is a solution of (2.7) if in addition it satisfies the boundary conditions. Notice that $\psi$ is defined in $\mathbb{R}$, then there is no need to impose any assumptions on the boundedness of $u^{\prime}$ in $[0, R]$. In fact, the structure of the equation implies a natural bound on $u^{\prime}$, as the following proposition evidences. 
Proposition 2.1. Assume $\left(\mathrm{h}_{1}\right)$. Then a positive function $u \in C^{1}([0, R])$ is a solution of (1.1) if and only if it is a solution of (2.7).

Proof. Let $u$ be a positive solution of (1.1). From $u(R)=0$ and $\left\|u^{\prime}\right\|_{\infty}<1$, we obtain the estimate $0 \leq u(r)<R$ for all $r \in[0, R]$, so

$$
\tilde{f}(r, u(r))=f(r, u(r)) \text { for a.e. } r \in[0, R] .
$$

Since $u^{\prime}(0)=0$, integrating the equation in (1.1) between 0 and a fixed $\left.r \in\right] 0, R[$, we get

hence

$$
\left|r^{N-1} \phi\left(u^{\prime}(r)\right)\right| \leq \int_{0}^{r} s^{N-1}|f(s, u)| d s,
$$

$$
\left|\phi\left(u^{\prime}(r)\right)\right| \leq \int_{0}^{r}\left(\frac{s}{r}\right)^{N-1}|f(s, u)| d s \leq\|\gamma\|_{L^{1}} .
$$

Therefore $\left\|u^{\prime}\right\|_{\infty} \leq \phi^{-1}\left(\|\gamma\|_{L^{1}}\right)$, which implies that

$$
\psi\left(u^{\prime}(r)\right)=\phi\left(u^{\prime}(r)\right) \quad \text { for all } r \in[0, R]
$$

and we conclude that $u$ is a positive solution of (2.7).

On the other hand, if $u$ is a positive solution of (2.7), arguing as above, we see that $\left\|u^{\prime}\right\|_{\infty} \leq \psi^{-1}\left(\|\gamma\|_{L^{1}}\right)$. In particular, we get $\left\|u^{\prime}\right\|_{\infty}<1$. Then, as before, $0 \leq u(r)<R$ for all $r \in[0, R]$, so

$$
\begin{aligned}
\phi\left(u^{\prime}(r)\right) & =\psi\left(u^{\prime}(r)\right) & & \text { for all } r \in[0, R], \\
f(r, u(r)) & =\widetilde{f}(r, u(r)) & & \text { for a.e. } r \in[0, R]
\end{aligned}
$$

and $u$ is a positive solution of (1.1).

This proposition allows to turn our attention to the search of positive solutions of (2.7). In this context, for simplicity of notation, we will always denote the modified function by $f$.

In order to introduce the variational formulation of problem (2.7), following [6], we define the space

$$
\left.\left.\mathcal{H}_{N-1}(0, R)=\left\{w \in W_{\mathrm{loc}}^{1,1}(] 0, R\right]\right): \int_{0}^{R} r^{N-1}\left|w^{\prime}\right|^{2} d r<+\infty \text { and } w(R)=0\right\} .
$$

Notice that $\mathcal{H}_{N-1}(0, R)$ is a Hilbert space with respect to the norm

$$
\|w\|_{R}=\left(\int_{0}^{R} r^{N-1}\left|w^{\prime}\right|^{2} d r\right)^{1 / 2}
$$

and the inclusion holds $\left.\left.\mathcal{H}_{N-1}(0, R) \subseteq C(] 0, R\right]\right)$.

Now we point out some properties of $\mathcal{H}_{N-1}(0, R)$, which play a central role in the theorem we are going to prove. 
Lemma 2.2. For any $p>(N-2) / 2$, there exists a constant $c=c(R, p)>0$ such that the estimate

$$
\left\|r^{p} u\right\|_{\infty} \leq c\|u\|_{R}
$$

holds for all $u \in \mathcal{H}_{N-1}(0, R)$.

Proof. If $N>2$, the result is exactly [6, Corollary 2]. For completeness we show that it also holds for $N=2$. Fix $p>0$ : for any $r \in] 0, R]$, we have

$$
\begin{aligned}
\left|r^{p} u(r)\right| & =\left|r^{p} \int_{r}^{R} u^{\prime} d s\right| \\
& \leq\left(\int_{r}^{R} \frac{r^{2 p}}{s} d s\right)^{1 / 2}\left(\int_{r}^{R} s\left|u^{\prime}\right|^{2} d s\right)^{1 / 2} \leq\left(r^{2 p} \log \left(\frac{R}{r}\right)\right)^{1 / 2}\|u\|_{R} .
\end{aligned}
$$

Now name $l:] 0, R] \rightarrow \mathbb{R}$ the function $l(y)=y^{2 p} \log (R / y)$. This function is continuous and bounded, so there exists a positive constant $c=c(R, p)$ such that $|l(y)| \leq c$ for all $y \in] 0, R]$. Then we get the conclusion.

Lemma 2.3. For any $q \in\left[2,2^{*}[\right.$, there exists a constant $d=d(R, q)>0$ such that the estimate

$$
\int_{0}^{R} r^{N-1}|u|^{q} d r \leq d\|u\|_{R}^{q}
$$

holds for all $u \in \mathcal{H}_{N-1}(0, R)$.

Proof. As before, we refer to [6, Proposition 3] for the case $N>2$. As for $N=2$, we apply Lemma 2.2 , with $p=1 / q$ and we get

$$
\int_{0}^{R} r|u|^{q} d r=\int_{0}^{R}\left(r^{1 / q}|u|\right)^{q} d r \leq R\left\|r^{1 / q} u\right\|_{\infty}^{q} \leq d\|u\|_{R}^{q},
$$

where $d=R \cdot c^{q}$ and $c$ is the constant in the statement of the previous lemma

In particular, we deduce that there exists a Poincaré-like constant $C_{P}=$ $C_{P}(R)>0$ such that

$$
\int_{0}^{R} r^{N-1}|u|^{2} d r \leq C_{P}\|u\|_{R}^{2}, \quad \text { for all } u \in \mathcal{H}_{N-1}(0, R) .
$$

We are now in position of defining the action functional associated with (2.7), that is, for $v \in \mathcal{H}_{N-1}(0, R)$,

$$
\mathcal{I}(v)=\int_{0}^{R} r^{N-1} \Psi\left(v^{\prime}\right) d r-\int_{0}^{R} r^{N-1} F(r, v) d r .
$$

In the rest of the section, we list some properties of problem (2.7). 
LEMMA 2.4. If $u \in \mathcal{H}_{N-1}(0, R)$ is a critical point of $\mathcal{I}$, then $u$ belongs to $C^{1}([0, R])$ and is a solution of $(2.7)$.

Proof. The proof closely follows the line of [6, Proposition 5]. Since $u \in$ $\left.\left.W_{\text {loc }}^{1,1}(] 0, R\right]\right)$ and $|\psi(s)| \leq \sigma|s|$ for all $s \in \mathbb{R}$, we see that the function $r^{N-1} \psi\left(u^{\prime}\right)$ belongs to $\left.\left.L_{\text {loc }}^{1}(] 0, R\right]\right)$. Moreover, by $\left(h_{1}\right)$, the function $r^{N-1} f(r, u) \in L^{1}(0, R)$. On the other hand, since $u$ is a critical point of $\mathcal{I}$ in $\mathcal{H}_{N-1}(0, R)$, it satisfies $u(R)=0$ and

$$
\int_{0}^{R} r^{N-1} \psi\left(u^{\prime}\right) v^{\prime} d r=\int_{0}^{R} r^{N-1} f(r, u) v d r
$$

for all $v \in C_{0}^{\infty}(0, R)$. Therefore, we infer that $\left.\left.r^{N-1} \psi\left(u^{\prime}\right) \in W_{\text {loc }}^{1,1}(] 0, R\right]\right)$ and the following equality holds

$$
-\left(r^{N-1} \psi\left(u^{\prime}\right)\right)^{\prime}=r^{N-1} f(r, u),
$$

almost everywhere in $] 0, R\left[\right.$. As a consequence, $\left.\left.u \in C^{1}(] 0, R\right]\right)$. It remains to study the behaviour of $u$ as $r$ tends to $0^{+}$. Set

$$
\Gamma(s)=\int_{0}^{s} \gamma(\xi) d \xi
$$

where $\gamma$ is defined in (2.3). Notice that, since $\gamma \in L^{1}(0, R)$, its primitive $\Gamma \in$ $W^{1,1}(0, R)$. Taking $0<r_{1}<r_{2} \leq R$ and integrating (2.9) between $r_{1}$ and $r_{2}$, we get

$$
\begin{aligned}
& \mid r_{2}^{N-1} \psi\left(u^{\prime}\left(r_{2}\right)\right)-r_{1}^{N-1} \psi\left(u^{\prime}\left(r_{1}\right)\right)\left|\leq \int_{r_{1}}^{r_{2}} r^{N-1}\right| f(r, u) \mid d r \\
& \leq r_{2}^{N-1} \int_{r_{1}}^{r_{2}} \gamma(r) d r=r_{2}^{N-1}\left(\Gamma\left(r_{2}\right)-\Gamma\left(r_{1}\right)\right) .
\end{aligned}
$$

The uniform continuity of $\Gamma$ implies that the function $r^{N-1} \psi\left(u^{\prime}\right)$ has finite limit as $r$ tends to $0^{+}$. In particular, the condition

$$
\int_{0}^{R} r^{N-1}\left|u^{\prime}\right|^{2} d r<+\infty, \quad \text { that is } \int_{0}^{R} r^{N-1}\left|\psi\left(u^{\prime}\right)\right|^{2} d r<+\infty,
$$

forces the limit to be 0 . Hence, for any fixed $r \in] 0, R]$ estimate $(2.10)$ yields

$$
\left|r^{N-1} \psi\left(u^{\prime}(r)\right)\right| \leq r^{N-1} \Gamma(r) .
$$

Observing that $|s| \leq|\psi(s)|$ for all $s \in \mathbb{R}$, we have

$$
\left|u^{\prime}(r)\right| \leq\left|\psi\left(u^{\prime}(r)\right)\right| \leq \Gamma(r)
$$

for all $r \in] 0, R]$. In particular, $u \in C^{1}([0, R])$, with $u^{\prime}(0)=0$. We can conclude in this way that $u$ is a solution of $(2.7)$. 
Lemma 2.5. Assume $\left(h_{1}\right)$ and let $f(r, 0) \geq 0$, for almost every $r \in[0, R]$. If $u \in C^{1}([0, R])$ is a nontrivial solution of

$$
\left.-\left(r^{N-1} \psi\left(u^{\prime}\right)\right)^{\prime}=r^{N-1} f(r, u) \quad \text { in }\right] 0, R[,
$$

and is satisfies $u(R)=0$, then $u$ is positive.

Proof. Multiplying equation (2.11) by $u^{-}$and integrating from 0 to $R$, we get

$$
-\int_{0}^{R}\left(r^{N-1} \psi\left(u^{\prime}\right)\right)^{\prime} u^{-} d r=\int_{0}^{R} r^{N-1} f(r, u) u^{-} d r .
$$

By the modification introduced in $(2.2), f(r, s) \geq 0$ for almost every $r \in[0, R]$ and every $s \leq 0$, so the right-hand side of (2.12)is nonnegative. Notice that the function $u^{-}$belongs to $W^{1,1}(0, R)$, then we can integrate by parts the left-hand side of (2.12) and, by the oddness of $\psi$, we have

$\int_{0}^{R}\left(r^{N-1} \psi\left(u^{\prime}\right)\right)^{\prime} u^{-} d r=-\int_{0}^{R} r^{N-1} \psi\left(u^{\prime}\right)\left(u^{-}\right)^{\prime} d r=\int_{0}^{R} r^{N-1} \psi\left(\left(u^{-}\right)^{\prime}\right)\left(u^{-}\right)^{\prime} d r$.

Therefore we get

$$
\int_{0}^{R} r^{N-1} \psi\left(\left(u^{-}\right)^{\prime}\right)\left(u^{-}\right)^{\prime} d r \leq 0 .
$$

Since $\psi$ is strictly increasing and $\psi(0)=0$, we have $\psi(s) s \geq 0$, for all $s \in \mathbb{R}$ and the equality holds if and only if $s=0$. Hence we can conclude that the nontrivial solution $u$ is such that $u^{-}=0$, that is, $u>0$.

\section{Main results}

Theorem 3.1. Assume $\left(\mathrm{h}_{1}\right)$,

$\left(\mathrm{h}_{2}\right)$ there exist $a, b$, with $0 \leq a<b \leq R$, such that

$$
\liminf _{s \rightarrow 0^{+}} \frac{F(r, s)}{s^{2}}>-\infty \quad \text { uniformly a.e. in }[a, b]
$$

$\left(\mathrm{h}_{3}\right)$ there exist $c, d$, with $a \leq c<d<b$, such that

$$
\limsup _{s \rightarrow 0^{+}} \int_{c}^{d} r^{N-1} \frac{F(r, s)}{s^{2}} d r=+\infty
$$

$\left(\mathrm{h}_{4}\right) f(r, 0) \geq 0$ for almost every $r \in[0, R]$,

$\left(\mathrm{h}_{5}\right) g:[0, R] \times \mathbb{R} \rightarrow \mathbb{R}$ satisfies the $L^{1}$-Carathéodory conditions,

and set

$$
G(r, s)=\int_{0}^{s} g(r, \xi) d \xi
$$


Assume further:

$\left(\mathrm{h}_{6}\right)$ there exists $w \in \mathcal{H}_{N-1}(0, R)$ such that $w>0,\left\|w^{\prime}\right\|_{\infty}<1$ and

$$
\int_{0}^{R} r^{N-1} G(r, w) d r>0
$$

$\left(\mathrm{h}_{7}\right) \lim \sup G(r, s) / s^{2} \leq 0$ uniformly almost everywhere in $[0, R]$,

$\left(\mathrm{h}_{8}\right) \liminf _{s \rightarrow 0^{+}} G(r, s) / s^{2}>-\infty$ uniformly almost everywhere in $[a, b]$, with a and $b$ defined in $\left(\mathrm{h}_{2}\right)$,

$\left(\mathrm{h}_{9}\right) g(r, 0)=0$ for almost every $r \in[0, R]$.

Then there exist $\mu^{*}>0$ and a function $\left.\left.\lambda:\right] \mu^{*},+\infty[\rightarrow] 0,+\infty\right]$ such that, for all $\mu>\mu^{*}$ and all $\left.\lambda \in\right] 0, \lambda(\mu)[$, the problem

$$
\left\{\begin{array}{l}
\left.-\left(\frac{r^{N-1} u^{\prime}}{\sqrt{1-\left|u^{\prime}\right|^{2}}}\right)^{\prime}=r^{N-1}(\lambda f(r, u)+\mu g(r, u)) \quad \text { in }\right] 0, R[ \\
u^{\prime}(0)=0, \quad u(R)=0
\end{array}\right.
$$

has at least three positive solutions.

Proof. Step 1. Variational setting of the problem. Following the procedure applied in Proposition 2.1, we replace $f$ and $g$ with positive functions, we still denote by $f$ and $g$, which satisfy all the assumptions of the theorem, and such that, for almost every $r \in[0, R], f(r, \cdot), g(r, \cdot)$ both coincide with the original functions in $[0, R], f(r, s)=0$ for $|s| \geq R+1, g(r, s)=0$ for $s \leq 0$ or $s \geq R+1$. Since the modified functions $f$ and $g$ vanish outside the rectangle $[0, R] \times[-(R+$ $1), R+1$ ] and satisfy the $L^{1}$-Carathéodory conditions, we can find constants $c_{f}, c_{g}>0$ such that

$$
\int_{0}^{R} r^{N-1}|F(r, v)| d r \leq c_{f} \quad \text { and } \quad \int_{0}^{R} r^{N-1} G(r, v) d r \leq c_{g}
$$

for all $v \in \mathcal{H}_{N-1}(0, R)$. Note that, by $\left(\mathrm{h}_{1}\right)$ and $\left(\mathrm{h}_{5}\right)$, there exists $\gamma \in L^{1}(0, R)$ such that

$$
\lambda|f(r, s)|+\mu|g(r, s)| \leq \gamma(r)
$$

for almost every $r \in[0, R]$ and every $s \in \mathbb{R}$. Without loss of generality, we can also suppose that $\phi\left(\left\|w^{\prime}\right\|_{\infty}\right)<\|\gamma\|_{L^{1}}$, where $w \in \mathcal{H}_{N-1}(0, R)$ is the function described in $\left(\mathrm{h}_{6}\right)$.

We define $\psi$ as in (2.4), $\Psi$ as in $(2.5)$ and $\mathcal{I}_{\lambda, \mu}: \mathcal{H}_{N-1}(0, R) \rightarrow \mathbb{R}$ by setting

$$
\mathcal{I}_{\lambda, \mu}(v)=\int_{0}^{R} r^{N-1} \Psi\left(v^{\prime}\right) d r-\lambda \int_{0}^{R} r^{N-1} F(r, v) d r-\mu \int_{0}^{R} r^{N-1} G(r, v) d r .
$$

for all $\lambda \geq 0, \mu \geq 0$, 
Step 2. Existence of a global minimizer. The functional $\mathcal{I}_{\lambda, \mu}$ is $C^{1}$ and weakly lower semicontinuous. Moreover, it is coercive and bounded from below: indeed, (2.6) and (3.2), we have

$$
\begin{aligned}
\mathcal{I}_{\lambda, \mu}(v) \geq \frac{1}{2} \int_{0}^{R} r^{N-1}\left|v^{\prime}\right|^{2} d r-\lambda \int_{0}^{R} r^{N-1} F(r, v) d r \\
-\mu \int_{0}^{R} r^{N-1} G(r, v) d r \geq \frac{1}{2}\|v\|_{R}^{2}-\left(\lambda c_{f}+\mu c_{g}\right)
\end{aligned}
$$

for all $v \in \mathcal{H}_{N-1}(0, R)$. Consequently, for each $\lambda \geq 0$ and $\mu \geq 0$ there exists $u_{1} \in \mathcal{H}_{N-1}(0, R)$ such that

$$
\mathcal{I}_{\lambda, \mu}\left(u_{1}\right)=\min _{v \in \mathcal{H}_{N-1}(0, R)} \mathcal{I}_{\lambda, \mu}(v)
$$

Take $\mu^{*}>0$ such that

$$
\int_{0}^{R} r^{N-1} \Phi\left(w^{\prime}\right) d r-\mu^{*} \int_{0}^{R} r^{N-1} G(r, w) d r+2 c_{f} \leq 0
$$

where $\Phi$ is defined in (2.1). Then if $\lambda \in[0,1]$ and $\mu>\mu^{*}$, we have

$$
\mathcal{I}_{\lambda, \mu}\left(u_{1}\right) \leq \mathcal{I}_{\lambda, \mu}(w)<-c_{f}<0
$$

which implies $u_{1} \neq 0$.

Step 3. Existence of a mountain pass critical point. We are now interested in searching a second critical point of $\mathcal{I}_{\lambda, \mu}$, using the mountain pass theorem (see [1]). Let us verify that the Palais-Smale condition holds. Take a Palais-Smale sequence $\left(u_{n}\right)_{n}$ in $\mathcal{H}_{N-1}(0, R)$. From (3.4) it follows that $\left(u_{n}\right)_{n}$ is bounded; this implies that there exists a subsequence, that we still denote by $\left(u_{n}\right)_{n}$, which weakly converges in $\mathcal{H}_{N-1}(0, R)$. Let $u$ be the limit of this sequence. Since $\left(\mathcal{I}_{\lambda, \mu}^{\prime}\left(u_{n}\right)[v]\right)_{n}$ converges to 0 , for all $v \in \mathcal{H}_{N-1}(0, R)$, choosing $v=u_{n}-u$, we get

$$
\begin{aligned}
\lim _{n \rightarrow+\infty}\left(\int_{0}^{R} r^{N-1} \psi\left(u_{n}^{\prime}\right)\left(u_{n}^{\prime}-u^{\prime}\right) d r\right. & -\lambda \int_{0}^{R} r^{N-1} f\left(r, u_{n}\right)\left(u_{n}-u\right) d r+ \\
& \left.-\mu \int_{0}^{R} r^{N-1} g\left(r, u_{n}\right)\left(u_{n}-u\right) d r\right)=0 .
\end{aligned}
$$

By definition of weak convergence in $\mathcal{H}_{N-1}(0, R)$ and (3.3), applying Lemma 2.2, we have

$$
\lim _{n \rightarrow+\infty} \int_{0}^{R} r^{N-1}\left(\lambda f\left(r, u_{n}\right)+\mu g\left(r, u_{n}\right)\right)\left(u_{n}-u\right) d r=0 .
$$

Then it follows that

$$
\lim _{n \rightarrow+\infty} \int_{0}^{R} r^{N-1} \psi\left(u_{n}^{\prime}\right)\left(u_{n}^{\prime}-u^{\prime}\right) d r=0 .
$$


Moreover, we have

$$
\lim _{n \rightarrow+\infty} \int_{0}^{R} r^{N-1} \psi\left(u^{\prime}\right)\left(u_{n}^{\prime}-u^{\prime}\right) d r=0,
$$

and then

$$
\lim _{n \rightarrow+\infty} \int_{0}^{R} r^{N-1}\left(\psi\left(u_{n}^{\prime}\right)-\psi\left(u^{\prime}\right)\right)\left(u_{n}^{\prime}-u^{\prime}\right) d r=0 .
$$

In order to conclude that $\lim _{n \rightarrow+\infty}\left\|u_{n}-u\right\|_{R}=0$, it suffices to observe that $\left(\psi\left(s_{1}\right)-\psi\left(s_{2}\right)\right)\left(s_{1}-s_{2}\right) \geq\left(s_{1}-s_{2}\right)^{2}$, for all $s_{1}, s_{2} \in \mathbb{R}$. This shows that the Palais-Smale condition holds.

On the other hand, let us check that, for sufficiently small positive $\lambda$, we are in presence of the mountain pass geometry around the origin. By assumptions $\left(\mathrm{h}_{5}\right)$ and $\left(\mathrm{h}_{7}\right)$ there exist $\vartheta>0$ and $\bar{\eta}>0$ such that $G(r, s) \leq \vartheta|s|^{2+\bar{\eta}}$ for almost every $r \in[0, R]$ and all $s \in \mathbb{R}$. We can now state that, for any given $\eta \in] 0, \bar{\eta}]$, the inequality

$$
\int_{0}^{R} r^{N-1} G(r, v) d r \leq \vartheta(R+1)^{\bar{\eta}} \int_{0}^{R} r^{N-1}|v|^{2+\eta} d r
$$

holds for all $v \in \mathcal{H}_{N-1}(0, R)$. Indeed, from $\left(\mathrm{h}_{9}\right)$ and from the definition of the truncated function $g$, we have $G(r, s)=0$ for all $s \leq 0$ and $G(r, s)=G(r, R+1)$ for all $s \geq R+1$. Therefore, for any $\eta \in[0, \bar{\eta}]$

$$
\begin{aligned}
\int_{0}^{R} r^{N-1} G(r, v) d r & =\int_{0}^{R} r^{N-1} G\left(r, v^{+} \wedge(R+1)\right) d r \\
& \leq \vartheta \int_{0}^{R} r^{N-1}\left|v^{+} \wedge(R+1)\right|^{2+\bar{\eta}} d r \\
& \leq \vartheta(R+1)^{2+\bar{\eta}} \int_{0}^{R} r^{N-1}\left(\frac{v^{+}}{R+1} \wedge 1\right)^{2+\bar{\eta}} d r \\
& \leq \vartheta(R+1)^{2+\bar{\eta}} \int_{0}^{R} r^{N-1}\left(\frac{v^{+}}{R+1} \wedge 1\right)^{2+\eta} d r \\
& =\vartheta(R+1)^{\bar{\eta}-\eta} \int_{0}^{R} r^{N-1}\left(v^{+} \wedge(R+1)\right)^{2+\eta} d r \\
& \leq \vartheta(R+1)^{\bar{\eta}} \int_{0}^{R} r^{N-1}|v|^{2+\eta} d r
\end{aligned}
$$

Fix $\eta \in] 0, \bar{\eta}]$ such that $2+\eta<2^{*}$, then by Lemma 2.3 there exists a constant $d_{\eta}>0$ such that

$$
\begin{aligned}
& \int_{0}^{R} r^{N-1} \Psi\left(v^{\prime}\right) d r-\mu \int_{0}^{R} r^{N-1} G(r, v) d r \\
& \geq \frac{1}{2}\|v\|_{R}^{2}-\mu \vartheta(R+1)^{\bar{\eta}} \int_{0}^{R} r^{N-1}|v|^{2+\eta} d r
\end{aligned}
$$




$$
\begin{aligned}
& \geq \frac{1}{2}\|v\|_{R}^{2}-\mu \vartheta(R+1)^{\bar{\eta}} d_{\eta}\|v\|_{R}^{2+\eta} \\
& \geq\|v\|_{R}^{2}\left(\frac{1}{2}-\mu \vartheta(R+1)^{\bar{\eta}} d_{\eta}\|v\|_{R}^{\eta}\right)
\end{aligned}
$$

for all $v \in \mathcal{H}_{N-1}(0, R)$. Now take $\left.\rho \in\right] 0,\|w\|_{R}[$ such that

$$
\frac{1}{2}-\mu \vartheta(R+1)^{\bar{\eta}} d_{\eta} r^{\eta}>0
$$

for all $r \in[0, \rho]$. Fix a constant $\lambda(\mu) \in] 0,1[$ such that

$$
r^{2}\left(\frac{1}{2}-\mu \vartheta(R+1)^{\bar{\eta}} d_{\eta} r^{\eta}\right)-\lambda(\mu) c_{f} \geq 0
$$

for all $r \in] 0, \rho]$ and pick any $\lambda \in] 0, \lambda(\mu)[$. By (3.7) and (3.8), we have

$$
\begin{aligned}
\mathcal{I}_{\lambda, \mu}(v) & =\int_{0}^{R} r^{N-1} \Psi\left(v^{\prime}\right) d r-\lambda \int_{0}^{R} r^{N-1} F(r, v) d r-\mu \int_{0}^{R} r^{N-1} G(r, v) d r \\
& \geq\|v\|_{R}^{2}\left(\frac{1}{2}-\mu \vartheta(R+1)^{\bar{\eta}} d_{\eta}\|v\|_{R}^{\eta}\right)-\lambda c_{f}>0
\end{aligned}
$$

for all $v \in \mathcal{H}_{N-1}(0, R)$ such that $\|v\|_{R}=\rho$. Since (3.6) also holds, by the mountain pass theorem we conclude that the functional $\mathcal{I}_{\lambda, \mu}$ has a critical point $u_{2}$, with $\mathcal{I}_{\lambda, \mu}\left(u_{2}\right)>0$ and then $u_{1} \neq u_{2}$.

Step 4. Existence of a local minimizer. We observe that there exists a local minimum point $u_{3}$ of $\mathcal{I}_{\lambda, \mu}$, with $\left\|u_{3}\right\|_{R}<\rho$. To verify that $u_{3} \neq 0$, let $\zeta \in$ $\mathcal{H}_{N-1}(0, R)$ be such that $0 \leq \zeta \leq 1$ in $[0, R], \zeta(r)=0$ for all $r \in[0, a] \cup[b, R]$, and $\zeta(r)=1$ for all $r \in[c, d]$. By assumptions $\left(\mathrm{h}_{2}\right),\left(\mathrm{h}_{3}\right)$ and $\left(h_{8}\right)$ there exist a constant $K>0$ and a strictly decreasing sequence $\left(c_{n}\right)_{n}$ satisfying

$$
\begin{gathered}
\lim _{n \rightarrow+\infty} c_{n}=0, \\
\lim _{n \rightarrow+\infty} \int_{c}^{d} r^{N-1} \frac{F\left(r, c_{n}\right)}{c_{n}^{2}} d r=+\infty, \\
F\left(r, c_{n} \zeta(r)\right) \geq-K c_{n}^{2} \zeta(r)^{2} \quad \text { for a.e. } r \in[a, b] \text { and all } n, \\
G\left(r, c_{n} \zeta(r)\right) \geq-K c_{n}^{2} \zeta(r)^{2} \quad \text { for a.e. } r \in[a, b] \text { and all } n .
\end{gathered}
$$

In particular we have $\left\|c_{n} \zeta\right\|_{R}<\rho$ for large $n$. Then we compute, using also (2.6) and (2.8),

$$
\begin{aligned}
\mathcal{I}_{\lambda, \mu}\left(c_{n} \zeta\right)= & \int_{0}^{R} r^{N-1} \Psi\left(c_{n} \zeta^{\prime}\right) d r \\
& -\lambda \int_{0}^{R} r^{N-1} F\left(r, c_{n} \zeta\right) d r-\mu \int_{0}^{R} r^{N-1} G\left(r, c_{n} \zeta\right) d r \\
\leq & \frac{\sigma}{2} \int_{0}^{R} r^{N-1}\left(c_{n} \zeta^{\prime}\right)^{2} d r
\end{aligned}
$$




$$
\begin{aligned}
& -\lambda \int_{a}^{b} r^{N-1} F\left(r, c_{n} \zeta\right) d r-\mu \int_{a}^{b} r^{N-1} G\left(r, c_{n} \zeta\right) d r \\
\leq & c_{n}^{2}\left(\frac{\sigma}{2}\|\zeta\|_{R}^{2}-\lambda \int_{c}^{d} r^{N-1} \frac{F\left(r, c_{n}\right)}{c_{n}^{2}} d r+C_{P}(\lambda+\mu) K\|\zeta\|_{R}^{2}\right)<0,
\end{aligned}
$$

for large $n$. Hence we have $\mathcal{I}_{\lambda, \mu}\left(u_{3}\right)<0$ and therefore $u_{3} \neq 0$. Finally we observe that, by (3.7) and (3.8), we have

$$
\mathcal{I}_{\lambda, \mu}\left(u_{3}\right) \geq\left\|u_{3}\right\|_{R}^{2}\left(\frac{1}{2}-\mu \vartheta(R+1)^{\bar{\eta}} d_{\eta}\left\|u_{3}\right\|_{R}^{\eta}\right)-\lambda c_{f}>-c_{f} .
$$

Since, by (3.6), $\mathcal{I}_{\lambda, \mu}\left(u_{1}\right)<-c_{f}$, we conclude that $u_{1} \neq u_{3}$.

Conclusion. The results contained in Lemmas 2.4 and 2.5 ensure that each critical point of $\mathcal{I}_{\lambda, \mu}$ is a nontrivial, therefore positive, solution of (3.1).

Proposition 3.2. Assume $\left(\mathrm{h}_{5}\right),\left(\mathrm{h}_{6}\right),\left(\mathrm{h}_{7}\right)$ and $\left(\mathrm{h}_{9}\right)$. Then there exists $\mu^{*}>0$ such that, for all $\mu>\mu^{*}$, the problem

$$
\left\{\begin{array}{l}
\left.-\left(\frac{r^{N-1} u^{\prime}}{\sqrt{1-\left|u^{\prime}\right|^{2}}}\right)^{\prime}=\mu r^{N-1} g(r, u) \quad \text { in }\right] 0, R[ \\
u^{\prime}(0)=0, \quad u(R)=0
\end{array}\right.
$$

has at least two positive solutions.

Proof. The functional associated with (3.12) is given by

$$
\mathcal{I}_{\mu}(v)=\int_{0}^{R} r^{N-1} \Psi\left(v^{\prime}\right) d r-\mu \int_{0}^{R} r^{N-1} G(r, v) d r
$$

for all $v \in \mathcal{H}_{N-1}(0, R)$. Taking $\mu^{*}>0$ as in (3.5), Steps 2 and 3 of the previous proof are still valid. This shows that for all $\mu>\mu^{*}$ there exist two nontrivial critical points $u_{1}$ and $u_{2}$ for $\mathcal{I}_{\mu}$, therefore they are positive solutions of (3.12)

Proposition 3.3. Assume $\left(\mathrm{h}_{5}\right),\left(\mathrm{h}_{6}\right)$ and $g(r, 0) \geq 0$ for almost every $r \in$ $[0, R]$. Then there exists $\mu^{*}>0$ such that, for all $\mu>\mu^{*}$, problem (3.12) has at least one positive solution.

Proof. As for Proposition 3.2, the proof essentially follows the ideas of Theorem 3.1. Taking $\lambda=0$ and $\mu>0$ in Step 2, we see that the functional $\mathcal{I}_{\mu}$ in (3.13) has a minimizer $u \in \mathcal{H}_{N-1}(0, R)$. In order to prove that $u \neq 0$, we observe that from $\left(\mathrm{h}_{6}\right)$ there exists $\mu^{*}>0$ such that

$$
\mathcal{I}_{\mu}(u) \leq \mathcal{I}_{\mu}(w)<0,
$$

for all $\mu>\mu^{*}$. Then $u$ is a positive solution of (3.12). 
Proposition 3.4. Assume $\left(\mathrm{h}_{1}\right),\left(\mathrm{h}_{2}\right),\left(\mathrm{h}_{3}\right)$ and $\left(\mathrm{h}_{4}\right)$. Then for all $\lambda>0$, the problem

$$
\left\{\begin{array}{l}
\left.-\left(\frac{r^{N-1} u^{\prime}}{\sqrt{1-\left|u^{\prime}\right|^{2}}}\right)^{\prime}=\lambda r^{N-1} f(r, u) \quad \text { in }\right] 0, R[, \\
u^{\prime}(0)=0, \quad u(R)=0
\end{array}\right.
$$

has at least one positive solution.

Proof. The functional associated with (3.14) is

$$
\mathcal{I}_{\lambda}(v)=\int_{0}^{R} r^{N-1} \Psi\left(v^{\prime}\right) d r-\lambda \int_{0}^{R} r^{N-1} F(r, v) d r
$$

for all $v \in \mathcal{H}_{N-1}(0, R)$. As in Steps 1 and 2 of the proof of Theorem 3.1, we obtain the existence of a global minimizer $u \in \mathcal{H}_{N-1}(0, R)$ of $\mathcal{I}_{\lambda}$. In order to conclude, we should prove that $u \neq 0$. Following the line of Step 4 , we take a function $\zeta \in \mathcal{H}_{N-1}(0, R)$, such that $0 \leq \zeta \leq 1$ in $[0, R], \zeta(r)=0$ for all $r \in[0, a] \cup[b, R]$ and $\zeta(r)=1$ for all $r \in[c, d]$. By $\left(\mathrm{h}_{2}\right)$ and $\left(\mathrm{h}_{3}\right)$ there exist a constant $K>0$ and a strictly decreasing sequence $\left(c_{n}\right)_{n}$ satisfying (3.9)-(3.11). Then we have

$$
\mathcal{I}_{\lambda}\left(c_{n} \zeta\right) \leq c_{n}^{2}\left(\frac{\sigma}{2}\|\zeta\|_{R}^{2}-\lambda \int_{c}^{d} r^{N-1} \frac{F\left(r, c_{n}\right)}{c_{n}^{2}} d r+\lambda C_{P} K\|\zeta\|_{R}^{2}\right) .
$$

Hence, for large $n$, we have $\mathcal{I}_{\lambda}(u) \leq \mathcal{I}_{\lambda}\left(c_{n} \zeta\right)<0$ and therefore $u$ is nontrivial. So, by Lemma $2.5, u$ is a positive solution of (3.14).

REMARK 3.5. The argument used in Step 3 to show the validity of (3.7) avoids to impose any restriction on the range of $p$ : whatever $p \in[1,+\infty[$ may be, the procedure described above can always be applied.

REMARK 3.6. Theorem 3.1 can also be stated in the case of an annular domain. One can show it following step-by-step the proof of the theorem produced here.

REmark 3.7. Assume that $f:[0, R] \times \mathbb{R} \rightarrow \mathbb{R}$ is continuous. Then any solution $u$ of $(1.1)$ belongs to $C^{2}([0, R])$. This can be seen as follows: let $u$ be a solution of $(1.1)$, then the function $h(\cdot)=f(\cdot, u(\cdot))$ is continuous in $[0, R]$. Integrating the equation in (1.1) between 0 and $r$, for any $r \in] 0, R]$, we obtain

$$
\phi\left(u^{\prime}(r)\right)=-\int_{0}^{r}\left(\frac{s}{r}\right)^{N-1} h(s) d s .
$$

Now we show that the function $\phi\left(u^{\prime}\right)$ belongs to $C^{1}([0, R])$. Obviously, we have $\left.\left.\phi\left(u^{\prime}\right) \in C^{1}(] 0, R\right]\right)$. Next we verify that the following limit holds

$$
\lim _{r \rightarrow 0^{+}} \frac{\phi\left(u^{\prime}(r)\right)}{r}=-\frac{h(0)}{N} .
$$


Fix $\varepsilon>0$. By continuity of $h$ at 0 , there exists $\delta>0$ such that $|h(s)-h(0)|<\varepsilon$ holds, for any $s \in[0, \delta[$. Taking $r \in] 0, \delta[$, we have

$$
\begin{aligned}
\left|\frac{h(0)}{N}+\frac{\phi\left(u^{\prime}(r)\right)}{r}\right| & =\left|\frac{h(0)}{N}-\frac{1}{r} \int_{0}^{r}\left(\frac{s}{r}\right)^{N-1} h(s) d s\right| \\
& =\left|\frac{1}{r^{N}} \int_{0}^{r} s^{N-1}(h(0)-h(s)) d s\right| \leq \frac{\varepsilon}{r^{N}} \int_{0}^{r} s^{N-1} d s=\frac{\varepsilon}{N},
\end{aligned}
$$

which confirms the validity of (3.15) and which proves that $\phi\left(u^{\prime}\right) \in C^{1}([0, R])$. Since $\phi^{-1} \in C^{1}(\mathbb{R})$, we conclude that $u \in C^{2}([0, R])$.

REMARK 3.8. Under the assumptions of Remark 3.7, any radial weak solution $v$ of $(1.2)$, with $v \in C^{1}\left(\overline{B_{R}}\right),\|\nabla v\|_{\infty}<1$, is of class $C^{2}$. In particular $v$ is a classical solution of (1.2). In fact, as $v$ is a weak solution of (1.2), it satisfies

$$
\int_{B_{R}} \frac{\nabla v \cdot \nabla w}{\sqrt{1-|\nabla v|^{2}}} d x=\int_{B_{R}} f(|x|, v) w d x
$$

for all $w \in C_{0}^{\infty}\left(B_{R}\right)$. Let us set $u(r)=v(x)$, for all $x \in \overline{B_{R}}$ and all $r \in[0, R]$ such that $r=|x|$ : Lemma 2.4 and Proposition 2.1 prove that $u$ is a solution of (1.1). Then, by Remark 3.7 we obtain that $u \in C^{2}([0, R])$. In order to conclude that $v \in C^{2}\left(\overline{B_{R}}\right)$, it is enough to observe that

$$
\partial_{x_{i}, x_{j}} v(0)=\delta_{i j} u^{\prime \prime}(0),
$$

for all $i, j \in\{1, \ldots, N\}$. Here $\delta_{i j}$ denotes as usual the Kronecker delta.

\section{Appendix}

Claim. Assume that $f: \mathbb{R} \rightarrow \mathbb{R}$ is of class $C^{1}$. Then any positive solution $u \in C^{2}\left(\overline{B_{R}}\right)$ of (1.3) is radially symmetric.

The proof is based on the results contained in [11]. Indeed, let $u \in C^{2}\left(\overline{B_{R}}\right)$ be a positive solution of (1.3) and let $L \in] 0,1\left[\right.$ be such that $\|\nabla u\|_{\infty}<L$ (such a constant exists by definition of solution of (1.3)). For simplicity we write the equation in (1.3) as

$$
-\operatorname{div}\left(a\left(|\nabla u|^{2}\right) \nabla u\right)=f(u),
$$

with $a:[0,1[\rightarrow \mathbb{R}$ given by

Easy computations yield

$$
a(\xi)=\frac{1}{\sqrt{1-\xi}} .
$$

$$
\operatorname{div}\left(a\left(|\nabla u|^{2}\right) \nabla u\right)=a\left(|\nabla u|^{2}\right) \sum_{i=1}^{N} \partial_{x_{i}, x_{i}} u+2 a^{\prime}\left(|\nabla u|^{2}\right) \sum_{i, j=1}^{N} \partial_{x_{i}} u \partial_{x_{j}} u \partial_{x_{i}, x_{j}} u .
$$


Let us consider the function $\mathbf{F}: \mathbb{R} \times \mathbb{R}^{N} \times \mathbb{R}^{N^{2}} \rightarrow \mathbb{R}$ given by

$$
\mathbf{F}\left(s, p_{i}, p_{i j}\right)=\bar{a}\left(|p|^{2}\right) \sum_{i=1}^{N} p_{i i}+2 \bar{a}^{\prime}\left(|p|^{2}\right) \sum_{i, j=1}^{N} p_{i j}+f(s) .
$$

Notice that the results in [11] cannot be applied directly. Therefore we introduce a modification $\bar{a}: \mathbb{R} \rightarrow \mathbb{R}$ of the function $a$, defined by

$$
\bar{a}(\xi)= \begin{cases}\alpha_{1}(\xi) & \text { if } \xi<0, \\ a(\xi) & \text { if } 0 \leq \xi \leq L^{2}, \\ \alpha_{2}(\xi) & \text { if } L^{2}<\xi<1, \\ c & \text { if } \xi \geq 1\end{cases}
$$

where the functions $\alpha_{1}, \alpha_{2}: \mathbb{R} \rightarrow \mathbb{R}$ and the constant $c$ are such that $\bar{a}$ belongs to $C^{\infty}(\mathbb{R})$, is increasing and positive. The function $u$ is also a positive solution of the modified problem

$$
\begin{cases}-\operatorname{div}\left(\bar{a}\left(|\nabla u|^{2}\right) \nabla u\right)=f(u) & \text { in } B_{R}, \\ u=0 & \text { on } \partial B_{R} .\end{cases}
$$

Notice that

$$
\operatorname{div}\left(\bar{a}\left(|\nabla u|^{2}\right) \nabla u\right)=\bar{a}\left(|\nabla u|^{2}\right) \sum_{i=1}^{N} \partial_{x_{i}, x_{i}} u+2 \bar{a}^{\prime}\left(|\nabla u|^{2}\right) \sum_{i, j=1}^{N} \partial_{x_{i}} u \partial_{x_{j}} u \partial_{x_{i}, x_{j}} u
$$

and there exists a constant $K>0$ such that $0 \leq \bar{a}^{\prime}(\xi)<K$ for all $\xi \in \mathbb{R}$. Let us consider the function $\overline{\mathbf{F}}: \mathbb{R} \times \mathbb{R}^{N} \times \mathbb{R}^{N^{2}} \rightarrow \mathbb{R}$ given by

$$
\overline{\mathbf{F}}\left(s, p_{i}, p_{i j}\right)=\bar{a}\left(|p|^{2}\right) \sum_{i=1}^{N} p_{i i}+2 \bar{a}^{\prime}\left(|p|^{2}\right) \sum_{i, j=1}^{N} p_{i j}+f(s) .
$$

The function $\overline{\mathbf{F}}$ satisfies all the assumptions in [11, Corollary 1, p. 221]. Then we can easily conclude that $u$ is symmetric with respect to any hyperplane passing through the origin, which means that $u$ is radially symmetric.

\section{REFERENCES}

[1] A. Ambrosetti and P.H. Rabinowitz, Dual variational methods in critical point theory and applications, J. Functional Analysis 14 (1973), 349-381.

[2] R. Bartnik and L. Simon, Spacelike hypersurfaces with prescribed boundary values and mean curvature, Comm. Math. Phys. 87 yr 1982/83, 131-152.

[3] C. Bereanu, P. Jebelean and J. Mawhin, Radial solutions for some nonlinear problems involving mean curvature operators in Euclidean and Minkowski spaces, Proc. Amer. Math. Soc. 137 (2009), 161-169.

[4] C. Bereanu, P. Jebelean and P. Torres, Positive radial solutions for Dirichlet problems with mean curvature operators in Minkowski space, J. Funct. Anal. 264 (2013), $270-287$. 
[5] C. Bereanu, P. Jebelean And P. Torres, Multiple positive radial solutions for a Dirichlet problem involving the mean curvature operator in Minkowski space, J. Funct. Anal. 265 (2013), 644-659.

[6] D. Bonheure, J.M. Gomes And L. Sanchez, Positive solutions of a second-order singular ordinary differential equation, Nonlinear Anal. 61 (2005), 1383-1399.

[7] I. Coelho, C. Corsato, F. Obersnel and P. Omari, Positive solutions of the Dirichlet problem for the one-dimensional Minkowski-curvature equation, Adv. Nonlinear Stud. 12 (2012), 621-638.

[8] C. Corsato, F. Obersnel, P. Omari and S. Rivetti, Positive solutions of the Dirichlet problem for the prescribed mean curvature equation in Minkowski space, J. Math. Anal. Appl. (2013), 227-239.

[9] C. Corsato, F. Obersnel, P. Omari and S. Rivetti, On the lower and upper solution method for the prescribed mean curvature equation in Minkowski space, Discrete Contin. Dyn. Syst. (2013), 159-169.

[10] C. Gerhardt, H-surfaces in Lorentzian manifolds, Comm. Math. Phys. 89 (1983), $523-553$.

[11] B. GidAs, W. Ni AND L. NiREnBERG, Symmetry and related properties via the maximum principle, Commun. Math. Phys. 68 (1979), 209-243.

[12] J. Mawhin, Radial solutions of Neumann problem for periodic perturbations of the mean extrinsic curvature operator, Milan J. Math. 79 (2011), 95-112.

ISABEL COELHo

Área Departamental de Matemática

Instituto Superior de Engenharia de Lisboa

Rua Conselheiro Emídio Navarro 1

1950-062 Lisboa, PORTUGAL

and

Département de Mathématique

Université Libre de Bruxelles

CP 214 Boulevard du Triomphe

1050 Bruxelles, BELGIUM

E-mail address: isabel.coelho@ulb.ac.be

Chiara Corsato And Sabrina Rivetti

Dipartimento di Matematica e Geoscienze

Università degli Studi di Trieste

Via A. Valerio 12/1

34127 Trieste, ITALY

E-mail address: chiara.corsato@phd.units.it, sabrina.rivetti@phd.units.it 